\title{
Generalized instantaneous modes in higher-order scalar-tensor theories
}

\author{
Antonio De Felice, ${ }^{1}$ David Langlois, ${ }^{2}$ Shinji Mukohyama, ${ }^{1,3,4}$ Karim Noui, ${ }^{4,2}$ and Anzhong Wang ${ }^{5,6}$ \\ ${ }^{1}$ Center for Gravitational Physics, Yukawa Institute for Theoretical Physics, \\ Kyoto University, 606-8502 Kyoto, Japan \\ ${ }^{2}$ Laboratoire Astroparticule et Cosmologie, CNRS, Université Paris Diderot Paris 7, 75013 Paris, France \\ ${ }^{3}$ Kavli Institute for the Physics and Mathematics of the Universe (WPI), \\ The University of Tokyo Institutes for Advanced Study, The University of Tokyo, \\ Kashiwa, Chiba 277-8583, Japan \\ ${ }^{4}$ Institut Denis Poisson (UMR CNRS 7013), Université François Rabelais, \\ Parc de Grandmont, 37200 Tours, France \\ ${ }^{5}$ Institute for Advanced Physics \& Mathematics, Zhejiang University of Technology, \\ Hangzhou 310032, China \\ ${ }^{6}$ GCAP-CASPER, Physics Department, Baylor University, Waco, Texas 76798-7316, USA
}

(Received 20 July 2018; published 15 October 2018)

\begin{abstract}
We consider higher-order, scalar-tensor theories which appear degenerate when restricted to the unitary gauge but are not degenerate in an arbitrary gauge. We dub them U-degenerate theories. We provide a full classification of theories that are either DHOST or U-degenerate and that are quadratic in second derivatives of the scalar field and discuss its extension to cubic and higher-order theories. Working with a simple example of U-degenerate theory, we find that, for configurations in which the scalar field gradient is time-like, the apparent extra mode in such a theory can be understood as a generalized instantaneous, or "shadowy" mode, which does not propagate. Appropriate boundary conditions, required by the elliptic nature of part of the equations of motion, lead to the elimination of the apparent instability associated with this extra mode.
\end{abstract}

DOI: 10.1103/PhysRevD.98.084024

\section{INTRODUCTION}

Scalar-tensor theories have always played a prominent role in providing alternative theories of gravity. During the last few years, special attention has been devoted to scalartensor theories whose Lagrangian contains second-order derivatives of a scalar field. An important requirement for such theories is the absence of any Ostrogradski ghost, i.e., an extra degree of freedom (d.o.f.) generically associated with higher time derivatives.

The absence of such a problematic extra mode is automatically guaranteed in degenerate higher-order, scalar-tensor (DHOST) theories introduced in [1,2], for which the degeneracy of the Lagrangian leads to constraints that eliminate this potential extra scalar d.o.f., even if the associated Euler-Lagrange equations are higher order. DHOST theories were explicitly constructed up to quadratic order in [1] (see also [3-5] for further details) and their full classification up to cubic order (in second derivatives) was completed in [6]. DHOST theories extend the class of

Published by the American Physical Society under the terms of the Creative Commons Attribution 4.0 International license. Further distribution of this work must maintain attribution to the author(s) and the published article's title, journal citation, and DOI. Funded by SCOAP.
Horndeski theories [7] and the (larger) class of beyond Horndeski theories [8,9] (another special subclass of DHOST theories was found in [10] via disformal transformations of the Einstein-Hilbert action).

In order to study higher-order, scalar-tensor (HOST) theories, it is often convenient to resort to the so-called unitary gauge, where the coordinates are chosen such that the scalar field is spatially uniform, i.e., with only a time dependence. In other words, the constant time hypersurfaces coincide with the constant scalar field hypersurfaces. This gauge choice is, of course, restricted to configurations where the gradient of the scalar field is timelike but this is a natural assumption in the cosmological context. In particular, the unitary gauge is a key ingredient of the effective description of modification of gravity, dark energy and inflation (see e.g., [11-17] and especially [18] devoted to DHOST theories).

For Beyond Horndeski theories, the counting of the number of d.o.f. was initially carried out via a Hamiltonian formulation in the unitary gauge $[8,9,19]$. Potential limitations of the unitary gauge were later pointed out in [20], where a Hamiltonian analysis valid in an arbitrary gauge was also presented for a particular Beyond Horndeski theory (which in fact is related to a Horndeski theory by a disformal transformation, according to the correspondence shown earlier in [9]). A Hamiltonian analysis in an 
arbitrary gauge, using explicitly the degeneracy of the Lagrangian, for all quadratic HOST (including DHOST) theories was subsequently given in [2].

A manifest pitfall of the unitary gauge is that there exist HOST theories which seem to be degenerate when written in the unitary gauge but are not degenerate in their fully covariant version and therefore are not DHOST theories. We will denote these theories U-degenerate. The purpose of the present work is to study this very special class of theories and better understand the number and role of the scalar d.o.f., from the point of view of the unitary gauge or from that of an arbitrary gauge.

In this work, we first present a systematic and simple way to classify all HOST theories that are either DHOST or U-degenerate. For quadratic theories (in second derivatives of the scalar field), we find that their Lagrangian $L$ can be written as the sum of a totally U-degenerate Lagrangian, by which we mean a Lagrangian whose kinetic terms (for the scalar and tensor modes) vanish in the unitary gauge, and another term that does not involve the metric curvature and can be written in a simple way that makes the degeneracy in the unitary gauge manifest. Both terms of the Lagrangian correspond to DHOST Lagrangians separately, but their sum is not a DHOST Lagrangian. We then generalize this result to Lagrangians that involve arbitrary powers of second derivatives $\phi_{\mu \nu} \equiv \nabla_{\nu} \nabla_{\mu} \phi$, starting with cubic theories. This provides a simple and systematic parametrization of theories that are either DHOST or U-degenerate.

Interestingly, U-degenerate HOST theories include as particular examples the khronometric theories discussed in $[21,22]$. For these theories, the extra mode that appears in the covariant formulation has been called "instantaneous mode." In the more general context that we consider here, the structure of the extra mode that appears is often more intricate than in the case of "instantaneous" modes. We will call this mode a "generalized instantaneous mode", or also "shadowy" mode for a shorter denomination.

The notion of generalized instantaneous or shadowy mode can easily be understood by considering the following example of a nondynamical Lagrangian in Minkowski spacetime,

$$
L[\psi]=\frac{1}{2} \psi \Delta \psi,
$$

where $\Delta$ is the Euclidean Laplacian operator. This Lagrangian leads to the Laplace equation $\Delta \psi=0$. In a different set of coordinates $\left(t^{\prime}, x^{\prime}, y^{\prime}, z^{\prime}\right)$, with $t^{\prime}=t+v x$ $(v \neq 0)$ and the same spatial coordinates, the Lagrangian for $\psi$ becomes

$$
\begin{aligned}
L[\psi] & =-\frac{1}{2}\left[\left(v \partial_{t^{\prime}} \psi+\partial_{x^{\prime}} \psi\right)^{2}+\left(\partial_{y^{\prime}} \psi\right)^{2}+\left(\partial_{z^{\prime}} \psi\right)^{2}\right] \\
& \ni-\frac{v^{2}}{2}\left(\partial_{t^{\prime}} \psi\right)^{2}
\end{aligned}
$$

which contains a kinetic term for $\psi$ (with a negative sign). In this new frame, the action seems to contain a dynamical d.o.f., which corresponds to a shadowy mode.

In order to better understand the "shadowy" mode that arises in U-degenerate HOST theories, we study in detail a simple toy-model. It is a higher-derivative scalar theory, inspired from U-degenerate HOST theories, which we study in a flat two-dimensional spacetime for simplicity. We consider some background solution and then make a linear perturbation analysis around this background solution in two different coordinate systems. In the first one, the background solution is only time-dependent, corresponding to the choice of the unitary gauge for the background. In the second one, the background solution is both time and space dependent, but the gradient of the background scalar field is still assumed to be timelike. We then identify, in both approaches, the d.o.f. of the system and study the correspondence between these two calculations.

We find that the extra mode (which appears when the background is time and space dependent) can be understood as a shadowy mode, which does not really propagate. Appropriate boundary conditions, ${ }^{1}$ required by the elliptic nature of part of the equations of motion, lead to the elimination of the apparent instability associated with this extra mode. Hence, our analysis in this simple toy-model reconciles the two seemingly contradictory points of view based on the unitary gauge and a nonunitary gauge. This toy model also illustrates that the unitary gauge (which can be used for configurations where the gradient of the scalar field is timelike) constitutes a convenient gauge choice, where the partially elliptic character of the equations of motion is more transparent and where it is thus easier to fix appropriate boundary conditions.

The paper is organized as follows. In Sec. II, we present the classification of HOST theories that are U-degenerate, first focussing on quadratic theories then extending our classification to higher order. In Sec. III, we study in detail a simple but illustrative example of U-degenerate theory in a two-dimensional Minkowski spacetime and analyse the number and nature of d.o.f., depending on the gauge chosen to describe the background solution. We conclude with a brief summary and a discussion. Some technical details are also given in the Appendices.

\section{U-DEGENERATE HOST THEORIES}

The goal of this section is to present a classification of U-degenerate HOST theories, i.e., higher-order, scalartensor (HOST) theories that are degenerate only in the

\footnotetext{
${ }^{1}$ As it will be clear in the paper, the boundary conditions are fixed at spatial infinity. Hence, we are implicitly assuming that the space-time can locally be decomposed as $\Sigma \times \mathbb{R}$ where the space slices $\Sigma$ are noncompact at any time. Nonetheless, one could generalize the analysis to compact hypersurfaces $\Sigma$ considering instead periodic boundary conditions for instance.
} 
unitary gauge. For this purpose, we actually provide a classification of theories that are either DHOST or U-degenerate, i.e., those that are degenerate at least in the unitary gauge. After a short review of DHOST theories, which enables us to introduce some useful notations, we classify HOST Lagrangians that are either DHOST or U-degenerate and that are quadratic in second derivatives $\phi_{\mu \nu}$. We then extend our classification to cubic theories and beyond in the last two subsections.

\section{A. DHOST theories}

We start with HOST theories whose Lagrangian is (at most) quadratic in the second derivatives of the scalar field. The action of these theories takes the form

$$
\begin{aligned}
S\left[g_{\mu \nu}, \phi\right]= & \int d^{4} x \sqrt{-g}\left[f_{2}(\phi, X) R+L_{\phi}^{(2)}\right. \\
& \left.+f_{0}(\phi, X)+f_{1}(\phi, X) \square \phi\right],
\end{aligned}
$$

where $R$ is the Ricci scalar, $f_{A}(\phi, X)$ are arbitrary functions of $\phi$ and $X \equiv \phi_{\mu} \phi^{\mu}$ with $\phi_{\mu} \equiv \nabla_{\mu} \phi$. The term $L_{\phi}^{(2)}$ denotes the most general minimal coupling Lagrangian quadratic in $\phi_{\mu \nu} \equiv \nabla_{\mu} \phi_{\nu}$ and is given by

$$
L_{\phi}^{(2)} \equiv \sum_{A} \alpha_{A}(\phi, X) L_{A}^{(2)}
$$

where $\alpha_{A}(\phi, X)$ are functions of $\phi$ and $X$, and the elementary quadratic Lagrangians $L_{A}^{(2)}$ are

$L_{1}^{(2)}=\phi_{\mu \nu} \phi^{\mu \nu}, \quad L_{2}^{(2)}=(\square \phi)^{2}, \quad L_{3}^{(2)}=\left(\phi^{\mu} \phi^{\nu} \phi_{\mu \nu}\right) \square \phi$,

$L_{4}^{(2)}=\left(\phi_{\mu \nu} \phi^{\nu} \phi^{\mu \sigma} \phi_{\sigma}\right), \quad L_{5}^{(2)}=\left(\phi^{\mu} \phi^{\nu} \phi_{\mu \nu}\right)^{2}$.

These theories can be extended to include cubic terms, by adding to the action (2.1) the terms

$\int d^{4} x \sqrt{-g}\left(f_{3}(\phi, X) \phi_{\mu \nu} G^{\mu \nu}+\sum_{A=1}^{10} b_{A}(\phi, X) L_{A}^{(3)}\right)$,

where the ten elementary cubic Lagrangians $L_{A}^{(3)}$ are [6]

$$
\begin{aligned}
& L_{1}^{(3)}=(\square \phi)^{3}, \quad L_{2}^{(3)}=(\square \phi) \phi_{\mu \nu} \phi^{\mu \nu}, \quad L_{3}^{(3)}=\phi_{\mu \nu} \phi^{\nu \rho} \phi_{\rho}^{\mu}, \\
& L_{4}^{(3)}=(\square \phi)^{2} \phi_{\mu} \phi^{\mu \nu} \phi_{\nu}, \quad L_{5}^{(3)}=\square \phi \phi_{\mu} \phi^{\mu \nu} \phi_{\nu \rho} \phi^{\rho}, \quad L_{6}^{(3)}=\phi_{\mu \nu} \phi^{\mu \nu} \phi_{\rho} \phi^{\rho \sigma} \phi_{\sigma}, \\
& L_{7}^{(3)}=\phi_{\mu} \phi^{\mu \nu} \phi_{\nu \rho} \phi^{\rho \sigma} \phi_{\sigma}, \quad L_{8}^{(3)}=\phi_{\mu} \phi^{\mu \nu} \phi_{\nu \rho} \phi^{\rho} \phi_{\sigma} \phi^{\sigma \lambda} \phi_{\lambda}, \\
& L_{9}^{(3)}=\square \phi\left(\phi_{\mu} \phi^{\mu \nu} \phi_{\nu}\right)^{2}, \quad L_{10}^{(3)}=\left(\phi_{\mu} \phi^{\mu \nu} \phi_{\nu}\right)^{3} .
\end{aligned}
$$

In general, these theories propagate two scalar modes in addition to the usual two tensorial modes, one of the two scalar modes being an Ostrogradsky mode. However, when the Lagrangian is degenerate (i.e., it admits at least one primary constraint in addition to the usual constraints associated with the diff-invariance), the theory propagates at most three d.o.f.: the extra constraints enable us to eliminate some d.o.f. The classification of degenerate theories up to cubic order has been completed in [6].

\section{B. Classification of U-degenerate quadratic Lagrangians}

In order to classify all Lagrangians that are either DHOST or U-degenerate, it is useful to start from the ADM decomposition of (2.1) in the unitary gauge, ignoring the $f_{0}$ and $f_{1}$ terms which do not play any role in the degeneracy. We thus write the four-dimensional metric in the form

$$
d s^{2}=-N^{2} d t^{2}+\gamma_{i j}\left(d x^{i}+N^{i} d t\right)\left(d x^{j}+N^{j} d t\right),
$$

where $N$ and $N^{i}$ are the lapse and shift, respectively, and $\gamma_{i j}$ is the three-dimensional metric on constant $t$ spatial hypersurfaces. In the following, a dot will denote a partial derivative with respect to the time coordinate $t$.

As shown in [2], the kinetic part of the $(3+1)$ decomposition of the action (2.1) can be written in the form

$S_{\text {kin }}=\int d t d^{3} x N \sqrt{\gamma}\left(\mathcal{A} \dot{A}_{*}^{2}+2 \mathcal{B}^{i j} \dot{A}_{*} K_{i j}+\mathcal{K}^{i j k l} K_{i j} K_{k l}\right)$,

where $K_{i j}$ is the extrinsic curvature tensor and

$$
A_{*} \equiv \frac{1}{N}\left(\dot{\phi}-N^{i} \partial_{i} \phi\right)
$$

In the unitary gauge (where $\partial_{i} \phi=0$ ), the coefficients that appear in (2.7) reduce to

$$
\begin{gathered}
\mathcal{A}_{\mathrm{U}}=\alpha_{1}+\alpha_{2}+\left(\alpha_{3}+\alpha_{4}\right) X_{U}+\alpha_{5} X_{U}^{2}, \\
\mathcal{B}_{\mathrm{U}}^{i j}=4 f_{2 X}+2 \alpha_{2}+\alpha_{3} X_{U},
\end{gathered}
$$

$\mathcal{K}_{\mathrm{U}}^{i j, k l}=\left(f_{2}-\alpha_{1} X_{U}\right) \gamma^{i(k} \gamma^{l) j}-\left(f_{2}-\alpha_{2} X_{U}\right) \gamma^{i j} \gamma^{k l}$, 
with $X_{\mathrm{U}} \equiv-A_{*}^{2}=-\dot{\phi}^{2} / N^{2}$, corresponding to the expression of $X$ in the unitary gauge. The full expressions of these coefficients in an arbitrary gauge can also be found in [2], but we will not need them here.

Let us first identify the Lagrangians that are nondynamical when restricted to the unitary gauge, i.e., for which all of the above coefficients vanish. As one can immediately see, this imposes four conditions on the six functions $f_{2}$ and $\alpha_{A}$. Thus, the family of Lagrangians which are nondynamical, i.e., totally U-degenerate, in the unitary gauge can be expressed in terms of only two free functions (four conditions for six initial free functions), for instance $f_{2}$ and $\alpha_{5}$, while the other four are determined by the relations

$\alpha_{1}=-\alpha_{2}=\frac{f_{2}}{X}, \quad \alpha_{3}=\frac{2}{X}\left(\frac{f_{2}}{X}-2 f_{2 X}\right)$,

$\alpha_{4}=\frac{2}{X}\left(2 f_{2 X}-\frac{f_{2}}{X}\right)-X \alpha_{5}$.

This means that the quadratic Lagrangians that are totally U-degenerate in the unitary gauge can explicitly be written in the form

$$
\begin{aligned}
L_{\mathrm{tUd}}\left[f_{2}, \alpha_{5}\right] \equiv & f_{2} R+\frac{f_{2}}{X}\left(L_{1}^{(2)}-L_{2}^{(2)}\right) \\
& +\frac{2}{X^{2}}\left(f_{2}-2 X f_{2 X}\right)\left(L_{3}^{(2)}-L_{4}^{(2)}\right) \\
& -\alpha_{5}\left(X L_{4}^{(2)}-L_{5}^{(2)}\right),
\end{aligned}
$$

where $f_{2}$ and $\alpha_{5}$ are free functions.

In order to classify all quadratic HOST theories that are U-degenerate, it is convenient to decompose any Lagrangian into a totally U-degenerate part (2.13), which includes the Ricci scalar term, and another part which depends only on the five elementary Lagrangians of (2.3). The total Lagrangian thus reads

$$
L=L_{\mathrm{tUd}}\left[f_{2}, 0\right]+\tilde{L}_{\phi},
$$

where $\tilde{L}_{\phi}$ is of the form (2.2). As already mentioned, the $f_{0}$ and $f_{1}$ terms are not taken into account here because they do not modify the degeneracy properties of the total Lagrangian.

Since the kinetic part of $L_{\mathrm{tUd}}\left[f_{2}, 0\right]$ vanishes in the unitary gauge, it is easy to see that any Lagrangian $L$ is U-degenerate if and only if the Lagrangian $\tilde{L}_{\phi}$ is also U-degenerate. Moreover, degeneracy of $\tilde{L}_{\phi}$ means that the kinetic part of the Lagrangian, in the unitary gauge, can be written in the form

$$
\tilde{L}_{\phi, \mathrm{kin}}=\hat{\mathcal{K}}_{\mathrm{U}}^{i j, k l}\left(K_{i j}+\sigma \gamma_{i j} \dot{A}_{*}\right)\left(K_{k l}+\sigma \gamma_{k l} \dot{A}_{*}\right),
$$

where

$$
\hat{\mathcal{K}}_{\mathrm{U}}^{i j, k l}=-X_{\mathrm{U}}\left(\alpha_{1} \gamma^{i(k} \gamma^{l) j}-\alpha_{2} \gamma^{i j} \gamma^{k l}\right),
$$

which corresponds to (2.11) with $f_{2}=0$, since $\tilde{L}_{\phi}$ does not contain any curvature term by construction. By expanding (2.15) and comparing with (2.7), one finds (by eliminating $\sigma$ ) that the U-degenerate form (2.15) is possible if and only if the functions $\alpha_{A}$ satisfy the relation

$$
\begin{aligned}
& 4\left(\alpha_{1}+3 \alpha_{2}\right)\left(\alpha_{1}+\alpha_{2}+X\left(\alpha_{3}+\alpha_{4}\right)+X^{2} \alpha_{5}\right) \\
& \quad=3\left(2 \alpha_{2}+X \alpha_{3}\right)^{2} .
\end{aligned}
$$

Not surprisingly, it coincides with the degeneracy condition in the unitary gauge, already derived in [1]. Note that, by definition, U-degenerate theories satisfy the condition (2.17) but not all three degenerate conditions obtained in [1].

The expression of the Lagrangian written in the unitary gauge can easily be "covariantized" by using the Stueckelberg trick (see the Appendix for the correspondence). One thus obtains, instead of the parametrization in terms of the functions $\alpha_{A}$, a parametrization of all U-degenerate theories in terms of the five functions $f_{2}$, $\kappa_{1}, \kappa_{2}, \sigma$ and $\alpha$, which depend on $X$ and $\phi$, with a Lagrangian of the form

$$
L=L_{\mathrm{tUd}}\left[f_{2}, \alpha\right]+\hat{\mathcal{K}}^{\mu \nu, \rho \sigma}\left(\phi_{\mu \nu}+\sigma Y g_{\mu \nu}\right)\left(\phi_{\rho \sigma}+\sigma Y g_{\rho \sigma}\right),
$$

where

$$
\begin{array}{rlrl}
\hat{\mathcal{K}}^{\mu \nu, \rho \sigma} & \equiv \kappa_{1} h^{\mu(\rho} h^{\nu) \sigma}+\kappa_{2} h^{\mu \nu} h^{\rho \sigma}, & h_{\mu \nu} \equiv g_{\mu \nu}-\frac{1}{X} \phi_{\mu} \phi_{\nu}, \\
Y & \equiv \phi^{\alpha} \phi_{\alpha \beta} \phi^{\beta} .
\end{array}
$$

This is, of course, compatible with the parametrization (2.14) in terms of six functions constrained by the single relation (2.17). To make the relationship between these two parametrizations explicit, let us expand (2.18) in terms of the elementary Lagrangians. One obtains

$$
\begin{aligned}
L= & f_{2} R+\left(\kappa_{1}+\frac{f_{2}}{X}\right) L_{1}^{(2)}+\left(\kappa_{2}-\frac{f_{2}}{X}\right) L_{2}^{(2)} \\
& +\left(2 \frac{f_{2}}{X^{2}}-4 \frac{f_{2 X}}{X}+2 \sigma \kappa_{1}+2\left[3 \sigma-\frac{1}{X}\right] \kappa_{2}\right) L_{3}^{(2)} \\
& +\left(\alpha+2 \frac{f_{2 X}}{X}-\frac{2 f_{2}}{X^{2}}-\frac{2}{X} \kappa_{1}\right) L_{4}^{(2)} \\
& +\left(-\frac{\alpha}{X}+\frac{2 f_{2 X}}{X^{2}}+\kappa_{1}\left[\frac{1}{X^{2}}+3 \sigma^{2}-\frac{2 \sigma}{X}\right]\right. \\
& \left.+\kappa_{2}\left[3 \sigma-\frac{1}{X}\right]^{2}\right) L_{5}^{(2)},
\end{aligned}
$$

and it is straightforward to check that this Lagrangian indeed satisfies the condition (2.17). The above Lagrangian 
includes both U-degenerate theories and DHOST theories, since the latter also satisfy the condition (2.17).

\section{Beyond quadratic order}

In order to classify all theories that are either DHOST or U-degenerate up to third order in second derivatives of $\phi$, one can follow the same strategy as in the previous section and first identify the theories that are totally U-degenerate, i.e., nondynamical in the unitary gauge. By using the ADM decomposition of HOST theories, up to cubic order, given in [18], one finds that all the kinetic terms vanish in the unitary gauge when the following eleven relations are satisfied by the functions $\alpha_{A}, b_{A}$ and $f_{A}$ :

$$
\begin{array}{lc}
b_{1}=b_{2}=b_{3}=0, & b_{4}=-b_{6}=-\frac{f_{3 X}}{X}, \quad b_{5}+X b_{9}=-\frac{2 f_{3 X}}{X}, \\
b_{7}+X b_{8}+X^{2} b_{10}=\frac{2 f_{3 X}}{X}, & \alpha_{1}=\frac{f_{2}}{X}+\frac{f_{3 \phi}}{2}, \quad \alpha_{2}=-\frac{f_{2}}{X}+\frac{f_{3 \phi}}{2}, \\
\alpha_{3}=\frac{2}{X}\left(\frac{f_{2}}{X}-\frac{f_{3 \phi}}{X}-2 f_{2 X}\right), & \alpha_{4}+X \alpha_{5}=\frac{2}{X}\left(2 f_{2 X}-\frac{f_{2}}{X}\right) .
\end{array}
$$

Since the initial Lagrangian depends on 17 functions, this implies that totally U-degenerate theories depend on 6 arbitrary functions that can be chosen to be $f_{3}, b_{8}, b_{9}, b_{10}$ for the cubic part, and $f_{2}, \alpha_{5}$, as before for the quadratic part, so that

$$
\begin{aligned}
& L_{\mathrm{tUd}}\left[f_{2}, f_{3}, \alpha_{5}, b_{8}, b_{9}, b_{10}\right] \\
& \quad=L_{\mathrm{tUd}}^{(2)}\left[f_{2}, f_{3}, \alpha_{5}\right]+L_{\mathrm{tUd}}^{(3)}\left[f_{3}, b_{8}, b_{9}, b_{10}\right],
\end{aligned}
$$

where, on the right-hand side, we have separated the terms that can be expressed in terms of the scalar curvature and of the quadratic Lagrangians (2.3), and those written in terms of $G^{\mu \nu} \phi_{\mu \nu}$ and (2.5). Note that the quadratic part depends on $f_{3}$ too, if $f_{3 \phi}$ is nonzero.

Similarly to the quadratic case discussed previously, all U-degenerate Lagrangians up to cubic order can be written in the form

$$
\begin{aligned}
L= & L_{\mathrm{tUd}}\left[f_{2}, f_{3}, \alpha_{5}, b_{8}, b_{9}, b_{10}\right] \\
& +\hat{\mathcal{K}}^{\mu \nu, \rho \sigma}\left(\phi_{\mu \nu}+\sigma Y g_{\mu \nu}\right)\left(\phi_{\rho \sigma}+\sigma Y g_{\rho \sigma}\right) \\
& +\hat{\mathcal{K}}_{3}^{\mu \nu, \rho \sigma, \alpha \beta}\left(\phi_{\mu \nu}+\sigma Y g_{\mu \nu}\right)\left(\phi_{\rho \sigma}+\sigma Y g_{\rho \sigma}\right)\left(\phi_{\alpha \beta}+\sigma Y g_{\alpha \beta}\right),
\end{aligned}
$$

where

$$
\begin{aligned}
\mathcal{K}_{3}^{\mu \nu, \rho \sigma, \alpha \beta} \equiv & \omega_{1} h^{\mu \nu} h^{\rho \sigma} h^{\alpha \beta}+\omega_{3}\left(h^{\nu(\rho} h^{\sigma)(\alpha} h^{\beta) \mu}+h^{\mu(\rho} h^{\sigma)(\alpha} h^{\beta) \nu}\right) \\
& +\omega_{2}\left(h^{\mu(\rho} h^{\sigma) \nu} h^{\alpha \beta}+h^{\alpha(\rho} h^{\sigma) \beta} h^{\mu \nu}+h^{\alpha(\mu} h^{\nu) \beta} h^{\rho \sigma}\right),
\end{aligned}
$$

with $\sigma$ and $\omega_{A}$ arbitrary functions of $\phi$ and $X$. One can show that the three parts of the Lagrangian (2.23) correspond separately to DHOST theories. In fact, the last two terms of (2.23), which do not depend on $f_{2}$ and $f_{3}$, correspond to any DHOST Lagrangian satisfying $f_{2}=f_{3}=0$. They have been classified in [6], but can also be written in this very simple form, parametrized by 10 arbitrary functions of $X$ and $\phi$, namely $\alpha_{5}, b_{8}, b_{9}, b_{10}, \kappa_{1}, \kappa_{2}, \omega_{1}, \omega_{2}, \omega_{3}$ and $\sigma$. One can verify that these Lagrangians indeed satisfy the degeneracy conditions presented in [6].

One can then generalize these results to parametrize U-degenerate theories with a Lagrangian $L$ that contains arbitrary powers of $\phi_{\mu \nu}$. Following (2.23), one writes $L$ as $L=L_{\mathrm{tUd}}+L_{\phi}$ where $L_{\mathrm{tUd}}$ is given by (2.22) and contains all the curvature terms, while $L_{\phi}$ is a degenerate Lagrangian obtained by combining $X_{\mu \nu} \equiv \phi_{\mu \nu}+\sigma Y g_{\mu \nu}$ with the projector $h_{\mu \nu}$,

$$
L_{\phi}=\mathcal{K}\left[\phi_{\mu \nu}+\sigma Y g_{\mu \nu}, h^{\rho \sigma}\right] .
$$

Formally, one can expand $\mathcal{K}$ as

$$
\mathcal{K}\left(X_{\mu \nu}\right)=\sum_{A} \mathcal{K}_{A}^{\mu_{1} \nu_{1}, \mu_{2} \nu_{2}, \ldots, \mu_{A} \nu_{A}} X_{\mu_{1} \nu_{1}} \cdots X_{\mu_{A} \nu_{A}},
$$

where $\mathcal{K}_{A}$ are tensors constructed from $h^{\mu \nu}$ only. Once again, let us stress that the general Lagrangians given above include both U-degenerate theories and DHOST theories.

\section{AN ILLUSTRATIVE EXAMPLE}

After having classified all U-degenerate theories in the previous section, we would like to better understand the number of d.o.f. present in these theories, as well as their nature. In particular, since U-degenerate theories are degenerate in the unitary gauge but nondegenerate in another gauge, one would naively expect the presence of a single scalar d.o.f. in the unitary gauge but the appearance of an extra scalar d.o.f. when working in another gauge. We would like to understand how these two seemingly contradictory points of view can be reconciled.

For simplicity, we are going to restrict our analysis to a very simple model, directly inspired from the classification 
of the previous section but for which we ignore the tensor d.o.f. to concentrate only on the scalar modes. Such a simple model is provided, for instance, by the totally Udegenerate Lagrangian $L_{\mathrm{tUd}}[0, \mu]$ defined in (2.13), where we choose $\mu$ to be constant, restricted to a Minkowski spacetime. This Lagrangian is however too simple in the sense that it does not contain any propagating d.o.f. in the unitary gauge, where it is totally degenerate. For this reason, we add to this Lagrangian a standard kinetic term, which guarantees the presence of a propagating d.o.f. in the unitary gauge.

We thus consider the following Lagrangian

$$
L=-\frac{1}{2} X-\mu\left(X L_{4}^{(2)}-L_{5}^{(2)}\right) \text {. }
$$

Since we do not consider the metric fluctuations, the kinetic part of the Lagrangian written in the ADM form (2.7) reduces, ignoring $f_{0}$ (in the present case, $-X / 2$ ), to

$$
L_{\mathrm{kin}}=\mathcal{A} \dot{A}_{*}^{2},
$$

where

$$
\mathcal{A}=\alpha_{1}+\alpha_{2}-\left(\alpha_{3}+\alpha_{4}\right) A_{*}^{2}+\alpha_{5} A_{*}^{4}=\mu\left(X A_{*}^{2}+A_{*}^{4}\right) .
$$

In the unitary gauge, $X=-A_{*}^{2}$ and $\mathcal{A}$ vanishes. However, in an arbitrary gauge, we find

$$
\mathcal{A}=\mu A_{*}^{2}\left(\partial_{i} \phi\right)^{2}
$$

which does not vanish in general. We would thus expect to find an extra mode in this case.

\section{A. Analysis in a unitary gauge background}

Assuming that the scalar field has a timelike spacetime gradient, we can work in the unitary gauge and consider the background field

$$
\bar{\phi}=t
$$

Considering the perturbed solution

$$
\phi=t+\chi(t, x)
$$

the Lagrangian quadratic in perturbations is given by

$$
\mathcal{L}_{0}=\frac{1}{2}\left(\dot{\chi}^{2}-\chi^{\prime 2}\right)+\mu \dot{\chi}^{\prime 2},
$$

and no second time derivative appears. This is to be expected since the background is in the unitary gauge.
The dispersion relation is

$$
\left(1+2 \mu k^{2}\right) \omega^{2}-k^{2}=0,
$$

which gives the two solutions

$$
\omega= \pm \frac{k}{\sqrt{2 \mu k^{2}+1}},
$$

corresponding to a single d.o.f. Here, as a boundary condition, we have implicitly assumed that the field does not diverge at spatial infinity so that $k$ is real.

\section{B. Analysis in a nonunitary gauge background}

We now consider a background solution of the form

$$
\bar{\phi}=t+\alpha x,
$$

which is also a solution of the equations of motion. If $\alpha \neq 0$, this background solution is not described in the unitary gauge since the scalar field has now an explicit spatial dependence.

We then consider the perturbed solution

$$
\phi=t+\alpha x+\chi(t, x) .
$$

Substituting into (3.1), one can derive the Lagrangian quadratic in $\chi$, which reads

$$
\begin{aligned}
\mathcal{L}_{\alpha}= & \frac{1}{2}\left(\dot{\chi}^{2}-\chi^{\prime 2}\right)+\mu\left[\alpha^{2}\left(\ddot{\chi}^{2}+\chi^{\prime \prime 2}\right)\right. \\
& \left.-2 \alpha\left(1+\alpha^{2}\right)\left(\ddot{\chi} \dot{\chi}^{\prime}+\chi^{\prime \prime} \dot{\chi}^{\prime}\right)+\left(1+4 \alpha^{2}+\alpha^{4}\right) \dot{\chi}^{\prime 2}\right] .
\end{aligned}
$$

One can immediately check that, when $\alpha=0$, one recovers the previous case (3.7).

By considering plane wave solutions of the equations of motion, of the form $\chi \propto \exp (-i \omega t+i k x)$, one obtains the dispersion relation

$$
\begin{gathered}
2 \alpha^{2} \mu \omega^{4}+4 \alpha\left(\alpha^{2}+1\right) k \mu \omega^{3}+\left(2\left(\alpha^{4}+4 \alpha^{2}+1\right) k^{2} \mu+1\right) \omega^{2} \\
+4 \alpha\left(\alpha^{2}+1\right) k^{3} \mu \omega+2 \alpha^{2} k^{4} \mu-k^{2}=0 .
\end{gathered}
$$

In contrast with (3.8), this dispersion relation is polynomial in $\omega$ up to fourth order, when $\alpha \neq 0$ (if $\alpha=0$, one recovers (3.8) obviously). This leads to four solutions for $\omega$ : two of them are real and we will denote them $\omega_{1}$ and $\omega_{2}$. The other two are complex conjugate, i.e., of the form

$$
\omega_{3,4}=\omega_{ \pm}=\omega_{r} \pm i \omega_{i} .
$$

The four solutions of the dispersion relation (3.13), for a particular choice of $\alpha$ and $\mu$, are plotted in Fig. 1. 


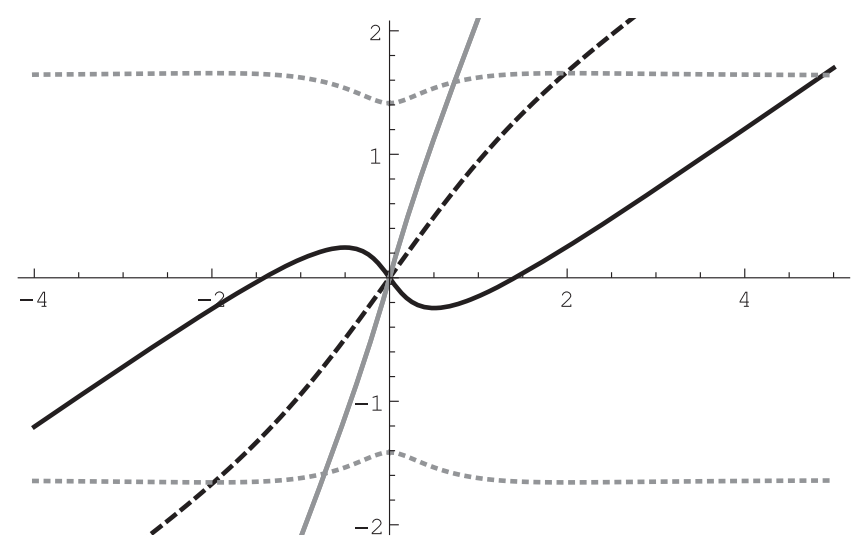

FIG. 1. Frequency $\omega$ as a function of $k$ for the four modes that appear in the nonunitary gauge: $\omega_{1}(k), \omega_{2}(k)$ correspond respectively to the dashed and continuous black curves. The real and imaginary parts of $\omega_{3}(k)$ and $\omega_{4}(k)$ are plotted respectively as continuous and dotted grey curves.

A priori, the fact that the equation of motion is fourth order indicates that four initial conditions need to be specified to evolve the system. At some initial time, say $t=0$, one needs as initial data the four functions $\chi(0, x)$, $\dot{\chi}(0, x), \ddot{\chi}(0, x), \dddot{\chi}(0, x)$, which can be assumed to be regular and to decay at spatial infinity (or even be nonzero only in a compact region of space).

In Fourier space, the equation for $\chi$ yields an ordinary differential equation for each Fourier mode $\chi(t, k)$, which is fourth order in time derivatives. This equation admits four independent particular solutions of the form $\chi(t, k) \propto e^{i \omega t}$, corresponding to the four solutions $\omega$ of the dispersion relation (3.13). As a consequence, the general solution can be written in the form

$$
\chi(t, k)=\sum_{A=1}^{4} u_{A}(k) e^{i \omega_{A}(k) t},
$$

where the coefficients $u_{A}(k)$ are determined from the initial conditions $\chi(0, x), \dot{\chi}(0, x), \ddot{\chi}(0, x), \dddot{\chi}(0, x)$, or equivalently $\chi^{(n)}(0, k)$ for $n=0,1,2,3$, by inverting the four relations

$$
\chi^{(n)}(0, k)=\sum_{A=1}^{4}(i \omega)^{n} u_{A}(k) .
$$

This yields

$$
u_{1}=\frac{\omega_{2} \omega_{3} \omega_{4} \chi(0)+i\left(\omega_{2} \omega_{3}+\omega_{3} \omega_{4}+\omega_{4} \omega_{2}\right) \dot{\chi}(0)-\left(\omega_{2}+\omega_{3}+\omega_{4}\right) \ddot{\chi}(0)-i \dddot{\chi}(0)}{\left(\omega_{2}-\omega_{1}\right)\left(\omega_{3}-\omega_{1}\right)\left(\omega_{4}-\omega_{1}\right)},
$$

and similar expressions for the other coefficients $u_{A}$, up to a permutation of the indices $A$.

Substituting these coefficients into (3.15), one obtains the full time evolution of $\chi(t, k)$, or equivalently $\chi(t, x)$ via inverse Fourier transform. In the generic case where $u_{3}$ and $u_{4}$ are nonzero, the imaginary part of $\omega_{3}$ and $\omega_{4}$ implies an exponential time evolution, thus signalling an apparent instability. As we shall see below, this instability can be avoided by taking into account appropriate boundary conditions, e.g., the regularity at spatial infinity.

\section{Comparison between the two approaches}

In this subsection, we discuss how the two previous analyses can be reconciled. In the following, we assume that $-1<\alpha<1$ so that the gradient of the background scalar field (3.10) is timelike.

First of all, let us note that the background solution (3.10), given in a nonunitary gauge, can also be described in a unitary gauge by using a new coordinate system, obtained via the boost transformation

$$
\begin{aligned}
& \tilde{t}=\tilde{T}(t, x) \equiv \frac{1}{\sqrt{1-\alpha^{2}}}(t+\alpha x), \\
& \tilde{x}=\tilde{X}(t, x) \equiv \frac{1}{\sqrt{1-\alpha^{2}}}(x+\alpha t) .
\end{aligned}
$$

In the coordinates $(\tilde{t}, \tilde{x})$, the scalar field (3.10) is given by $\bar{\phi}=\sqrt{1-\alpha^{2}} \tilde{t}$.

Accordingly, on substituting

$\omega=\frac{1}{\sqrt{1-\alpha^{2}}}(\tilde{\omega}-\alpha \tilde{k}), \quad k=\frac{1}{\sqrt{1-\alpha^{2}}}(\tilde{k}-\alpha \tilde{\omega})$,

which correspond to the (inverse) boost of the wave vector $(\tilde{\omega}, \tilde{k})$, into the dispersion relation (3.13) gives

$$
\left(1+2 \tilde{\mu} \tilde{k}^{2}\right) \tilde{\omega}^{2}-\tilde{k}^{2}=0, \quad \tilde{\mu} \equiv \mu\left(1-\alpha^{2}\right)^{2},
$$

which is of the form (3.8), with a rescaling of $\mu$ due to the fact that $\bar{\phi}$ is not strictly equal to $\tilde{t}$, but simply proportional to it.

Similarly, the equation of motion can be rewritten as

$$
\left[1-2 \mu\left(1-\alpha^{2}\right)^{2} \partial_{\tilde{x}}^{2}\right] \partial_{\tilde{t}}^{2} \chi-\partial_{\tilde{x}}^{2} \chi=0,
$$

where

$$
\partial_{\tilde{t}} \equiv \frac{1}{\sqrt{1-\alpha^{2}}}\left(\partial_{t}-\alpha \partial_{x}\right), \quad \partial_{\tilde{x}} \equiv \frac{1}{\sqrt{1-\alpha^{2}}}\left(\partial_{x}-\alpha \partial_{t}\right),
$$


and the perturbation $\chi=\chi(T(\tilde{t}, \tilde{x}), X(\tilde{t}, \tilde{x}))$ is now viewed as a function of $(\tilde{t}, \tilde{x})$ with

$X(\tilde{t}, \tilde{x})=\frac{1}{\sqrt{1-\alpha^{2}}}(\tilde{x}-\alpha \tilde{t}), \quad T(\tilde{t}, \tilde{x})=\frac{1}{\sqrt{1-\alpha^{2}}}(\tilde{t}-\alpha \tilde{x})$.

In contrast with the equation of motion written in the original coordinate system, the equation (3.21) is only second order in time derivatives. One can easily decompose this fourth-order equation of motion into two second-order equations, one hyperbolic and the other elliptic,

$$
\begin{gathered}
\left(\partial_{\tilde{t}}^{2}-\partial_{\tilde{x}}^{2}\right) \chi=\psi, \\
\left(\partial_{\tilde{x}}^{2}-\kappa^{2}\right) \psi=-\partial_{\tilde{x}}^{4} \chi,
\end{gathered}
$$

where

$$
\kappa=\frac{1}{\sqrt{2 \mu}} \frac{1}{1-\alpha^{2}} .
$$

Hereafter we assume $\mu>0$ so that $\kappa$ is real and positive. The second equation (3.25) can be easily integrated on a hypersurface where $\tilde{t}$ is constant (i.e., $t+\alpha x$ is constant) provided a boundary condition for $\psi$ is properly specified at infinity. Hence, there is clearly no instability in this frame.

To understand how the two approaches are related, let us express one of the complex frequency modes found above, i.e.,

$$
\chi_{\mathrm{sh}}(t, x)=e^{i(\omega t-k x)}, \quad \text { with } \quad \omega=\omega_{r} \pm i \omega_{i},
$$

in terms of the coordinates $(\tilde{t}, \tilde{x})$ gives

$$
\begin{aligned}
\chi_{\mathrm{sh}}(\tilde{t}, \tilde{x})= & \exp \left\{i\left[\frac{\left(\omega_{r}+\alpha k\right) \tilde{t}-\left(k+\alpha \omega_{r}\right) \tilde{x}}{\sqrt{1-\alpha^{2}}}\right]\right\} \\
& \times e^{\mp \frac{\omega_{i}}{\sqrt{1-\alpha^{2}}} \tilde{t}} e^{ \pm \frac{\alpha \omega_{i}}{\sqrt{1-\alpha^{2}}} \tilde{x}} .
\end{aligned}
$$

This shows that these modes diverge at spatial infinity in the new coordinate system. This explains why these modes do not appear when one starts the analysis around a unitary gauge background and demands the regularity of the initial data at spatial infinity.

Imposing appropriate boundary conditions for the elliptic equation (3.25), for instance that the acceptable solutions should be well-behaved at spatial infinity, eliminates the complex frequency modes (3.28). In the description (3.15), such boundary conditions would impose that $u_{3}=u_{4}=0$. In terms of initial conditions, this implies that the second and third order derivatives $\chi^{(2)}(0, k)$ and $\chi^{(3)}(0, k)$ are not independent but are instead fixed in terms of $\chi(0, k)$ and $\dot{\chi}(0, k)$. Explicitly, one finds

$$
\begin{gathered}
\chi^{(2)}(0, k)=\omega_{1} \omega_{2} \chi(0, k)+i\left(\omega_{1}+\omega_{2}\right) \dot{\chi}(0, k), \\
\chi^{(3)}(0, k)=i \omega_{1} \omega_{2}\left(\omega_{1}+\omega_{2}\right) \chi(0, k) \\
-\left(\omega_{1}^{2}+\omega_{1} \omega_{2}+\omega_{2}^{2}\right) \dot{\chi}(0, k) .
\end{gathered}
$$

One can check that the single d.o.f. that appears with a unitary gauge background automatically verifies the above constraints. Such a mode is of the form

$$
\begin{aligned}
\chi_{u}(t, x) & =e^{i(\tilde{\omega} \tilde{T}(t, x)-\tilde{k} \tilde{X}(t, x))} \\
& =\exp \left\{i\left[\frac{(\tilde{\omega}-\alpha \tilde{k}) t-(\tilde{k}-\alpha \tilde{\omega}) x}{\sqrt{1-\alpha^{2}}}\right]\right\},
\end{aligned}
$$

where $\tilde{\omega}$ satisfies the unitary gauge dispersion relation (3.8). By computing the time derivatives of $\chi_{u}$, one can verify that the conditions (3.29) and (3.30) are indeed satisfied.

In summary, we have found that arbitrary initial conditions, defined in some Lorentz frame where the background field is space dependent, generically lead to the presence of an apparent exponential instability. However, this instability is eliminated by imposing appropriate boundary conditions required to solve the elliptic part of the equations of motion.

\section{Green's function and emergence of a light cone at long distance}

In this section, we study further the dynamics of the perturbation $\chi$ in both coordinate systems. Let us start considering the equation of motion for $\chi$ in the $(\tilde{t}, \tilde{x})$ coordinate systems written in the form (3.24) and (3.25). To integrate the first equation (3.24) explicitly, it is useful to introduce the Green's function $G\left(\tilde{x}, \tilde{x}^{\prime}\right)$, defined by

$$
\left(\partial_{\tilde{x}}^{2}-\kappa^{2}\right) G\left(\tilde{x}, \tilde{x}^{\prime}\right)=\delta\left(\tilde{x}-\tilde{x}^{\prime}\right) .
$$

If we require the regularity condition at infinity

$$
\lim _{\tilde{x} \rightarrow \pm \infty}\left|G\left(\tilde{x}, \tilde{x}^{\prime}\right)\right|<\infty
$$

the Green's function (3.32) is given by

$$
G\left(\tilde{x}, \tilde{x}^{\prime}\right)=-\frac{1}{2 \kappa} e^{-\kappa\left|\tilde{x}-\tilde{x}^{\prime}\right|}
$$

As a consequence, the solution to (3.25) is

$\psi(\tilde{t}, \tilde{x})=\frac{1}{2 \kappa} \int d \tilde{y} \frac{\partial^{4}}{\partial \tilde{y}^{4}} \chi(T(\tilde{t}, \tilde{y}), X(\tilde{t}, \tilde{y})) e^{-\kappa|\tilde{x}-\tilde{y}|}$.

Substituting this solution back to (3.24), one obtains an equation for $\chi$ that includes only second order time derivatives. Obviously, $\psi$ represents the "shadowy" mode and the typical length scale of the "shadow" is $1 / \kappa$. In the 
limit $\kappa \rightarrow \infty$, the length of the "shadow" vanishes and there is no shadowy mode, as one can see directly from the Lagrangian (3.12).

From the previous analysis, we show that (with appropriate boundary conditions) the equation for $\chi$ in the $(\tilde{t}, \tilde{x})$ coordinate system reduces to

$$
\left(\partial_{\tilde{t}}^{2}-\partial_{\tilde{x}}^{2}\right) \chi=\frac{1}{2 \kappa} \int d \tilde{y} \frac{\partial^{4}}{\partial \tilde{y}^{4}} \chi(T(\tilde{t}, \tilde{y}), X(\tilde{t}, \tilde{y})) e^{-\kappa|\tilde{x}-\tilde{y}|} .
$$

Thus, the perturbation $\chi$ is uniquely determined by the datas of $\chi$ and $\partial_{\tilde{t} \chi}$ on a constant $\tilde{t}$ hypersurface, say $\tilde{t}=0$.

Now, when one considers the equation of motion in the $(t, x)$ coordinate system, one might wonder whether the values of $\chi$ and $\partial_{t} \chi$ on a constant $t$ hypersurface can uniquely determine the evolution of the system or not. To answer this question, we first reformulate (3.36) in the $(t, x)$ coordinate system as follows,

$$
\begin{gathered}
\left(\partial_{t}^{2}-\partial_{x}^{2}\right) \chi=\psi(t, x) \quad \text { with } \\
\psi(t, x)=\frac{1}{2 \kappa} \int d \tilde{y} \frac{\partial^{4}}{\partial \tilde{y}^{4}} \chi(T(\tilde{T}(t, x), \tilde{y}), X(\tilde{T}(t, x), \tilde{y})) \\
\times e^{-\kappa|\tilde{X}(t, x)-\tilde{y}|},
\end{gathered}
$$

where the functions $T, \tilde{T}, X$ and $\tilde{X}$ were given in (3.18) and (3.23). We will argue that the answer is positive, at least for $\kappa L \gg 1$, where $L$ is the length scale of interest which characterizes the variations of $\chi$ in space. For $\kappa L \gg 1$, we also argue that the concept of the light cone emerges.

The absolute value of the Green's function (3.34) has the maximum $(2 \kappa)^{-1}$ at $\tilde{x}=\tilde{x}^{\prime}$ and decays exponentially away from it. Hence, if the length scale $L$ is sufficiently longer than $1 / \kappa$ then, as one can easily confirm for each Fourier mode, (3.38) implies that $\psi$ scales as

$$
|\psi| \sim \mathcal{O}(\epsilon) \times\left|\partial_{X}^{2} \chi\right| \ll\left|\partial_{X}^{2} \chi\right|
$$

where we have introduced the small bookkeeping parameter $\epsilon=1 /(\kappa L)$. Therefore, at the lowest order in $\epsilon,(3.24)$ reduces to

$$
\left(\partial_{t}^{2}-\partial_{x}^{2}\right) \chi \simeq 0
$$

which gives an approximate solution $\chi \simeq \chi^{(0)}(t, x)$ from the initial values of $\chi$ and $\partial_{t} \chi$ on a hypersurface of constant $t$. For this approximate solution, the concept of the light cone makes sense (as we recover the usual d'Alembert equation).

Furthermore, one can systematically improve the approximation by expanding $\chi$ and $\psi$ in powers of $\epsilon$ as

$$
\chi=\chi^{(0)}+\chi^{(1)}+\chi^{(2)}+\cdots, \quad \psi=\psi^{(1)}+\psi^{(2)}+\cdots,
$$

where $\chi^{(n)}=\mathcal{O}\left(\epsilon^{n}\right)$ and $\psi^{(n)}=\mathcal{O}\left(\epsilon^{n}\right)$. Substituting these expansions in (3.37) and (3.38), at the lowest order in $\epsilon$, one recovers (3.40) for $\chi^{(0)}$ and

$$
\begin{aligned}
\psi^{(1)}(t, x)= & \frac{1}{2 \kappa} \int d \tilde{y} \frac{\partial^{4}}{\partial \tilde{y}^{4}} \chi^{(0)}(T(\tilde{T}(t, x), \tilde{y}), X(\tilde{T}(t, x), \tilde{y})) \\
& \times e^{-\kappa|\tilde{X}(t, x)-\tilde{y}|} .
\end{aligned}
$$

Suppose that the initial condition for $\chi$ is specified on an initial surface at $t_{0}$ as $\left(\chi\left(t_{0}, x\right), \partial_{t} \chi\left(t_{0}, x\right)\right)=\left(\chi_{0}(x), \chi_{1}(x)\right)$. One can easily solve (3.40) for $\chi^{(0)}$ with the initial condition given by $\left(\chi^{(0)}\left(t_{0}, x\right), \partial_{t} \chi^{(0)}\left(t_{0}, x\right)\right)=\left(\chi_{0}(x), \chi_{1}(x)\right)$, and obtain a solution $\chi^{(0)}(t, x)$ for all $(t, x)$. One can then calculate the right hand side of (3.42) to give $\psi^{(1)}(t, x)$ for all $(t, x)$. The leading correction to $\chi^{(0)}$ is given by solving the $\mathcal{O}(\epsilon)$ part of (3.37), namely

$$
\left(\partial_{t}^{2}-\partial_{x}^{2}\right) \chi^{(1)}=\psi^{(1)},
$$

with the initial condition $\chi^{(1)}\left(t_{0}, x\right)=\partial_{t} \chi^{(1)}\left(t_{0}, x\right)=0$. Higher-order corrections are also calculable in a similar way. The derivative (or long-distance) expansion (3.41) is expected to converge as far as $\epsilon \ll 1$.

We thus conclude that for $\mu>0$ and under the appropriate boundary condition, the values of $\chi$ and $\partial_{t} \chi$ on a surface of constant $t$ (instead of $\tilde{T}(t, x)$ constant) uniquely determines the evolution of the system as far as the length scale of interest is sufficiently longer than $1 / \kappa$. Moreover, in this limit, since $\chi^{(0)}$ gives a good approximation to the full solution $\chi$ and the concept of lightcone makes sense for $\chi^{(0)}$, we also conclude that the concept of lightcone emerges at long distances. In summary, if we are interested in physics at length scales sufficiently longer than the length of the "shadow" then the "shadowy" mode is invisible and the evolution of the system appears to be Lorentz-invariant.

\section{CONCLUSION}

We have studied higher-order, scalar-tensor theories that are not DHOST theories but are nevertheless degenerate when restricted to the unitary gauge. These theories, which we have dubbed U-degenerate, appear to contain one more dynamical d.o.f. in their covariant formulation than when restricted to the unitary gauge.

In the first part of the present work, we have shown how the class of theories that are either DHOST or U-degenerate can be systematically classified. We have found that quadratic theories of this class can be described by a Lagrangian that depends on five arbitrary functions [see Eq (2.20)], obtained by combining two DHOST 
Lagrangians: the first includes the curvature term (and is totally U-degenerate), the second can be written in a simple form where the degeneracy is manifest. We have then extended this description to theories that are cubic and higher order. Note that all our general Lagrangians that describe this class of theories also include as particular cases DHOST theories, since the latter automatically satisfy the unitary-gauge degeneracy condition, as a consequence of the full system of degeneracy conditions.

In the second part of this article, we have tried to reconcile the apparently contradictory points of view when the background scalar field (whose gradient is assumed to be timelike) is described in the unitary gauge or in a different gauge, by studying a simple toy model where the tensor modes, i.e., gravity, are ignored. In this model, we have found that the extra d.o.f. that appears in a nonunitary gauge can be understood as a generalized instantaneous mode, or "shadowy mode," which does not propagate. Indeed, this extra mode is governed by an elliptic equation, which is manifest in the unitary gauge (although somewhat obscured by the mixing of time and space in another gauge). Imposing appropriate boundary conditions, namely regularity at spatial infinity, leads to the elimination of the apparent instability in a nonunitary gauge. In this sense, the fact that the system in the unitary gauge seems to contain one less dynamical d.o.f. than in another gauge is due to the fact that the boundary conditions are already implemented implicitly in the unitary gauge, whereas they need to be taken into account explicitly in the other gauges.

Beyond the particular example we have studied, our analysis strongly suggests that U-degenerate theories, when the scalar field gradient is timelike and with appropriate boundary conditions, propagate a single scalar d.o.f., while the extra d.o.f., the shadowy mode, is nondynamical.

This would mean that, within these conditions, U-degenerate theories are safe from Ostrogradski instabilities and therefore worth exploring phenomenologically. The behavior of U-degenerate theories might differ from that of DHOST theories, ${ }^{2}$ and we plan to investigate their potentially new features in the future. It would also be very interesting to extend our analysis to the case where the tensor d.o.f. are taken into account, studying for example the linear perturbations about a nonisotropic cosmological background.

Another important issue related to the presence of the shadowy/instantaneous mode is the existence of black holes. In khronometric theories, it was shown that black holes still exist, but their boundaries are now universal horizons [22]. Recently, such black holes have been studied intensively $[23,24]$, and it would be very interesting to

\footnotetext{
${ }^{2}$ For linear cosmological perturbations, one can find in [18] the quadratic action for the physical scalar d.o.f. when taking into account only the unitary degeneracy condition.
}

investigate the existence, formation and thermodynamics of black holes in U-degenerate theories.

\section{ACKNOWLEDGMENTS}

K. N. and A. W. wish to warmly thank the YITP in Kyoto (where this article was initiated and later completed) for its hospitality. K. N. also wants to thank Y. Masahide for very interesting discussions on this topic during his stay at the Tokyo Institute of Technology. D. L. would like to thank the Yukawa Institute for Theoretical Physics at Kyoto University, where this work was completed during the YITP symposium YKIS2018a "General Relativity-The Next Generation" and the workshop YITP-T-17-02 "Gravity and Cosmology 2018." We also thank Gilles Esposito-Farese for very instructive discussions on this topic. A. D. F. was supported by JSPS KAKENHI Grants No. 16K05348 and No. 16H01099. The work of S. M. was supported by the Japan Society for the Promotion of Science (JSPS) Grants-in-Aid for Scientific Research (KAKENHI) No. 17H02890, No. 17H06359, and No. $17 \mathrm{H} 06357$ and by the World Premier International Research Center Initiative (WPI), MEXT, Japan. The work of A.W. is supported in part by the National Natural Science Foundation of China (NNSFC), Grants No. 11375153 and No. 11675145.

\section{APPENDIX A: INSTANTANEOUS MODES IN KHRONOMETRIC THEORIES}

Instantaneous modes have been introduced in the context of massive gravity in [25], and later on considered in $[21,22]$ in the context of khronometric theories which are simple examples of higher-order, scalar-tensor theories. In the latter case, the notion of instantaneous modes has been defined at the perturbative level about a homogeneous background.

Indeed, if one considers the dynamics of a small perturbation of the scalar field (the khronon), $\phi=t+\chi$ where $\chi$ is the perturbation about the solution $\phi=t$, on a fixed Minkowski background $g_{\mu \nu}=\eta_{\mu \nu}$, one easily sees that the quadratic action for $\chi$ is higher order in space derivatives only,

$$
S_{k h r}^{(2)}[\chi]=\int d^{4} x\left[\alpha\left(\partial_{i} \dot{\chi}\right)^{2}+\beta(\Delta \chi)^{2}\right],
$$

where $\alpha$ and $\beta$ can be reduced to nonzero constants (not functions) for our discussion here. The corresponding dispersion relation

$$
\alpha \omega^{2} k^{2}+\beta k^{4}=\alpha k^{2}\left(\omega^{2}-c_{s}^{2} k^{2}\right)=0,
$$

shows that the action describes a single mode propagating with a finite velocity $c_{s}^{2}=-\beta / \alpha$. However, one can interpret the presence of higher spatial derivatives in the equations of motion as the signature of a second mode 
propagating with an infinite speed: this is the reason why such a mode has been said to be instantaneous in [21]. Obviously, these instantaneous modes are simple examples of our shadowy modes.

\section{APPENDIX B: A COVARIANT FORM FOR THE TOTALLY DEGENERATE ACTION}

This section aims at formulating the Lagrangian (2.13) in a covariant form. For that purpose, we start using the (scalar) Gauss-Codazzi relation,

$$
R={ }^{3} R[h]+K_{\mu \nu} K^{\mu \nu}-K^{2}-2 \nabla_{\mu}\left(\mathbf{a}^{\mu}-K n^{\mu}\right),
$$

which links the four-dimensional Ricci scalar $R$ (associated to the metric $g_{\mu \nu}$ ) with the three-dimensional Ricci scalar ${ }^{3} R[h]$ associated to the three-dimensional spatial metric

$$
h_{\mu \nu} \equiv g_{\mu \nu}-\frac{1}{X} \phi_{\mu} \phi_{\nu}
$$

In (B1), the normal unit vector is given by

$$
n_{\mu} \equiv \frac{\phi_{\mu}}{\sqrt{-X}}
$$

from which we easily deduce the components of the acceleration vector $\mathbf{a}_{\mu}$ and of the fundamental two-form $K_{\mu \nu}$

$$
\begin{gathered}
\mathbf{a}_{\mu}=n^{\nu} \nabla_{\nu} n_{\mu}=-\frac{1}{X} h_{\mu \alpha} \phi^{\alpha \nu} \phi_{\nu} \\
=-\frac{1}{X}\left[\phi^{\nu} \phi_{\mu \nu}-\frac{1}{X}\left(\phi^{\alpha} \phi_{\alpha \beta} \phi^{\beta}\right) \phi_{\mu}\right], \\
K_{\mu \nu}=h_{\mu}^{\alpha} h_{\nu}^{\beta} \nabla_{\alpha} n_{\beta}=\frac{1}{\sqrt{-X}}\left[\phi_{\mu \nu}+\frac{1}{X^{2}}\left(\phi^{\alpha} \phi_{\alpha \beta} \phi^{\beta}\right) \phi_{\mu} \phi_{\nu}\right. \\
\left.-\frac{1}{X} \phi^{\beta}\left(\phi_{\mu} \phi_{\nu \beta}+\phi_{\nu} \phi_{\mu \beta}\right)\right] .
\end{gathered}
$$

Replacing these expressions in the (scalar) Gauss-Codazzi relation (B1), one easily shows, after an immediate calculation, that $L_{\mathrm{tUd}}\left[f_{2}, \alpha_{5}\right]$ can be expressed as

$$
\begin{aligned}
L_{\mathrm{tUd}}\left[f_{2}, \alpha_{5}\right]= & f_{2}{ }^{3} R[h]+\alpha \mathbf{a}^{2}+2 f_{2 \phi} h^{\mu \nu} \phi_{\mu \nu} \\
& +\nabla_{\mu}\left[\frac{2 f_{2}}{X}\left(\phi_{\nu} \phi^{\mu \nu}-\phi^{\mu} \square \phi\right)\right],
\end{aligned}
$$

where $\mathbf{a}^{2} \equiv \mathbf{a}_{\mu} \mathbf{a}^{\mu}$, and $\alpha \equiv-X^{3} \alpha_{5}$ is a function independent of $f_{2}$.

The expression (B6) is interesting because it shows explicitly (and in a covariant way) that $L_{\mathrm{tUd}}$ does not contain any second time derivatives of the scalar field. Second derivatives are space-like only. First of all, the original four-dimensional Ricci scalar combines with the
Lagrangians $L_{A}^{(2)}$ in order to reduce to the threedimensional Ricci scalar of $h_{\mu \nu}$, which is the first term in (B6), plus a small number of additional terms at the end of the calculation. Then, the second term in (B6), constructed from the acceleration vector, can be easily reformulated as follows:

$$
\alpha \mathbf{a}^{2}=\frac{\alpha}{4 X^{2}}\left(\partial_{\alpha} X\right) h^{\alpha \beta}\left(\partial_{\beta} X\right)=\left(\partial_{\alpha} F\right) h^{\alpha \beta}\left(\partial_{\beta} F\right),
$$

where $F(\phi, X)$ is a function of $\phi$ and $X$ such that $F_{X}^{2}=\alpha /\left(4 X^{2}\right)$. Even though $F$ contains time derivatives of $\phi$ via $X$, (B7) does not produce higher time derivatives, because only space derivatives of $F$ are present. Finally, the third term in (B6) involves also first time derivatives of $\phi$ only which appear via the Christoffel symbol $\Gamma_{\mu \nu}^{\rho}$ of the metric $g_{\mu \nu}$ according to

$$
h^{\mu \nu} \phi_{\mu \nu} \ni-h^{\mu \nu} \Gamma_{\mu \nu}^{0} \dot{\phi}
$$

Of course, we disregard the last term in (B6) which is an irrelevant total derivative.

\section{APPENDIX C: COUNTING DEGREES OF FREEDOM}

In this section, we count the number of d.o.f. of the totally degenerate theory (2.13) expressed in the unitary gauge thanks to a Hamiltonian analysis. To do so, we first rewrite the action (2.13) as follows

$$
\int d^{4} x \sqrt{\gamma}\left[f(N)^{3} R+\beta(N) \gamma^{i j} \dot{\gamma}_{i j}+\tilde{N}^{i} \partial_{i} N\right],
$$

where

$$
\begin{aligned}
f(N) & \equiv N f_{2}(N), \quad \beta(N) \equiv-\frac{f_{2 \phi}(N)}{N}, \\
\tilde{N}^{i} & \equiv 2 \frac{\partial \beta}{\partial N} N^{i}+\frac{\alpha_{5}}{N^{7}} \gamma^{i j} \partial_{j} N .
\end{aligned}
$$

For simplicity we have omitted to mention explicitly the time dependence of the functions in the Lagrangian. When $(\partial \beta / \partial N) \neq 0$ (what we assume here), one can change the variable $N^{i}$ by $\tilde{N}^{i}$. Integrating out this new variable, one obtains that $N$ is a function of time only, and then the action (C1) is shown to be equivalent to

$$
\int d^{4} x \sqrt{\gamma}\left[f(t)^{3} \mathrm{R}+\beta(t) \gamma^{i j} \dot{\gamma}_{i j}\right],
$$

where we have used the notation $f(t)$ for $f(t, N(t))$ (same thing for $\beta(t)$ ).

To start the Hamiltonian analysis, one introduces the 6 pairs of conjugate variables $\left(\gamma_{i j}, p^{k l}\right)$ with the Poisson bracket 


$$
\left\{\gamma_{i j}(\vec{x}), p^{k l}(\vec{y})\right\}=\delta_{(i}^{k} \delta_{j)}^{l} \delta^{3}(\vec{x}-\vec{y}),
$$

which satisfy the 6 primary constraints

$$
\chi^{i j} \equiv p^{i j}-\beta(t) \sqrt{\gamma} \gamma^{i j} \approx 0 .
$$

To go further, it is very useful to decompose the family of primary constraints into two independent sets $\left(\chi^{i j}\right)=$ $\left(\chi_{\|}^{i}, \chi_{\perp}^{i}\right)$ where $\chi_{\|}^{i}$ are the 3 longitudinal components of the constraints

$$
\chi_{\|}^{i} \equiv D_{j}\left(\frac{\chi^{i j}}{\sqrt{\gamma}}\right)=D_{j}\left(\frac{p^{i j}}{\sqrt{\gamma}}\right),
$$

and $\left(\chi_{\perp}^{i}\right)$ are the 3 transverse components. Thus, the total Hamiltonian reads

$$
H_{\mathrm{tot}}=\int d^{3} x \sqrt{\gamma}\left[-f(t)^{3} R+\lambda_{i} \chi_{\perp}^{i}+\mu_{i} \chi_{\|}^{i}\right],
$$

where $\lambda_{i}$ and $\mu_{i}$ are Lagrange multipliers which enforce the primary constraints. It is easy to see that $\chi_{\|}^{i} \approx 0$ are always conserved under time evolution whereas the conservation of $\chi_{\perp}^{i} \approx 0$ leads to 3 secondary constraints $\varphi_{i} \approx 0$. To see this in indeed the case, let us remark that

$$
\dot{\chi}_{i j}=\frac{\partial \chi_{i j}}{\partial t}+\left\{\chi_{i j}, H_{\mathrm{tot}}\right\}=f(t) G^{i j}-\dot{\beta}(t) \gamma^{i j} \approx 0,
$$

where $G^{i j}$ are the component of the Einstein tensor associated to $\gamma_{i j}$. Now, it becomes obvious (due to the conservation of $G^{i j}$ ) that $\dot{\chi}_{\|}^{i} \approx 0$ with no conditions, and only three components of $\dot{\chi}_{i j}$ are nonvanishing, which leads to three secondary constraints.

The Dirac algorithm closes here (there is no tertiary constraints) with 9 constraints in total: $\chi_{\|}^{i} \approx 0$ are in fact first class (and they are associated to the invariance of the theory under spatial diffeomorphisms); the six remaining constraints form a set of second class constraints. As we started with six pairs of variables, we end up with $[6-3-6 / 2]=0$ d.o.f.

In the special case where $(\partial \beta / \partial N)=0$, which means that $\beta$ depends on $t$ only, the action (C1) reduces to

$S=\int d^{4} x \sqrt{\gamma}\left[f(N)^{3} \mathrm{R}+\beta(t) \gamma^{i j} \dot{\gamma}_{i j}+\tilde{\alpha}(N) \gamma^{i j} \partial_{i} N \partial_{j} N\right]$,

where $\tilde{\alpha}(N)=\alpha_{5}(N) / N^{7}$. To make the Hamiltonian analysis, we start now with 7 pairs of conjugate variables

$$
\begin{aligned}
\left\{\gamma_{i j}(\vec{x}), p^{k l}(\vec{y})\right\} & =\delta_{(i}^{k} \delta_{j)}^{l} \delta^{3}(\vec{x}-\vec{y}), \\
\{N(x), \pi(y)\} & =\delta^{3}(\vec{x}-\vec{y}),
\end{aligned}
$$

which satisfy the seven primary constraints

$$
\chi^{i j} \equiv p^{i j}-\beta(t) \gamma^{i j} \approx 0, \quad \pi \approx 0 .
$$

The analysis of the constraints $\chi^{i j}$ is exactly the same as the previous case. Concerning the new constraint $\pi \approx 0$, its time evolution leads to the secondary constraint

$$
\mathcal{H} \equiv \frac{\delta S}{\delta N} \approx 0
$$

which is nothing but the Euler-Lagrange equation for the lapse $N$. There are no tertiary constraints and we end up with three first-class constraints (associated to the invariance under space diffeomorphisms) together with eight second-class constraints. As we have started with seven pairs of conjugate variables, here again we conclude that the theory has no d.o.f.
[1] D. Langlois and K. Noui, Degenerate higher derivative theories beyond Horndeski: Evading the Ostrogradski instability, J. Cosmol. Astropart. Phys. 02 (2016) 034.

[2] D. Langlois and K. Noui, Hamiltonian analysis of higher derivative scalar-tensor theories, J. Cosmol. Astropart. Phys. 07 (2016) 016.

[3] M. Crisostomi, K. Koyama, and G. Tasinato, Extended scalar-tensor theories of gravity, J. Cosmol. Astropart. Phys. 04 (2016) 044.

[4] C. de Rham and A. Matas, Ostrogradsky in theories with multiple fields, J. Cosmol. Astropart. Phys. 06 (2016) 041.
[5] J. Ben Achour, D. Langlois, and K. Noui, Degenerate higher order scalar-tensor theories beyond Horndeski and disformal transformations, Phys. Rev. D 93, 124005 (2016).

[6] J. Ben Achour, M. Crisostomi, K. Koyama, D. Langlois, K. Noui, and G. Tasinato, Degenerate higher order scalartensor theories beyond Horndeski up to cubic order, J. High Energy Phys. 12 (2016) 100.

[7] G. W. Horndeski, Second-order scalar-tensor field equations in a four-dimensional space, Int. J. Theor. Phys. 10, 363 (1974).

[8] J. Gleyzes, D. Langlois, F. Piazza, and F. Vernizzi, Healthy Theories Beyond Horndeski, Phys. Rev. Lett. 114, 211101 (2015). 
[9] J. Gleyzes, D. Langlois, F. Piazza, and F. Vernizzi, Exploring gravitational theories beyond Horndeski, J. Cosmol. Astropart. Phys. 02 (2015) 018.

[10] M. Zumalacárregui and J. García-Bellido, Transforming gravity: From derivative couplings to matter to second-order scalar-tensor theories beyond the Horndeski Lagrangian, Phys. Rev. D 89, 064046 (2014).

[11] N. Arkani-Hamed, H.-C. Cheng, M. A. Luty, and S. Mukohyama, Ghost condensation and a consistent infrared modification of gravity, J. High Energy Phys. 05 (2004) 074.

[12] N. Arkani-Hamed, P. Creminelli, S. Mukohyama, and M. Zaldarriaga, Ghost inflation, J. Cosmol. Astropart. Phys. 04 (2004) 001.

[13] P. Creminelli, M. A. Luty, A. Nicolis, and L. Senatore, Starting the universe: Stable violation of the null energy condition and non-standard cosmologies, J. High Energy Phys. 12 (2006) 080.

[14] C. Cheung, P. Creminelli, A. L. Fitzpatrick, J. Kaplan, and L. Senatore, The effective field theory of inflation, J. High Energy Phys. 03 (2008) 014.

[15] G. Gubitosi, F. Piazza, and F. Vernizzi, The effective field theory of dark energy, J. Cosmol. Astropart. Phys. 02 (2013) 032 .

[16] J. Gleyzes, D. Langlois, F. Piazza, and F. Vernizzi, Essential building blocks of dark energy, J. Cosmol. Astropart. Phys. 08 (2013) 025.
[17] J. Gleyzes, D. Langlois, and F. Vernizzi, A unifying description of dark energy, Int. J. Mod. Phys. D 23, 1443010 (2014).

[18] D. Langlois, M. Mancarella, K. Noui, and F. Vernizzi, Effective description of higher-order scalar-tensor theories, J. Cosmol. Astropart. Phys. 05 (2017) 033.

[19] C. Lin, S. Mukohyama, R. Namba, and R. Saitou, Hamiltonian structure of scalar-tensor theories beyond Horndeski, J. Cosmol. Astropart. Phys. 10 (2014) 071.

[20] C. Deffayet, G. Esposito-Farese, and D. A. Steer, Counting the degrees of freedom of generalized Galileons, Phys. Rev. D 92, 084013 (2015).

[21] D. Blas, O. Pujolas, and S. Sibiryakov, Models of nonrelativistic quantum gravity: The good, the bad and the healthy, J. High Energy Phys. 04 (2011) 018.

[22] D. Blas and S. Sibiryakov, Horava gravity versus thermodynamics: The black hole case, Phys. Rev. D 84, 124043 (2011).

[23] P. Berglund, J. Bhattacharyya, and D. Mattingly, Towards Thermodynamics of Universal Horizons in Einstein-æther Theory, Phys. Rev. Lett. 110, 071301 (2013).

[24] A. Wang, Hořava gravity at a Lifshitz point: A progress report, Int. J. Mod. Phys. D 26, 1730014 (2017).

[25] G. Gabadadze and L. Grisa, Lorentz-violating massive gauge and gravitational fields, Phys. Lett. B 617, 124 (2005). 